\title{
A Robust Longitudinal Control Strategy of Platoons under Model Uncertainties and Time Delays
}

\author{
Na Chen $\left(\mathbb{D},{ }^{1}\right.$ Meng Wang $\mathbb{D}^{1},{ }^{1}$ Tom Alkim, ${ }^{2}$ and Bart van Arem $\mathbb{D}^{1}$ \\ ${ }^{1}$ Department of Transport and Planning, Delft University of Technology, Stevinweg 1, 2628 CN Delft, Netherlands \\ ${ }^{2}$ Rijkswaterstaat, Ministry of Infrastructure and Water Management, Utrecht, Netherlands \\ Correspondence should be addressed to Na Chen; na.chen@tudelft.nl
}

Received 1 September 2017; Accepted 20 February 2018; Published 15 April 2018

Academic Editor: Wai Yuen Szeto

Copyright (c) $2018 \mathrm{Na}$ Chen et al. This is an open access article distributed under the Creative Commons Attribution License, which permits unrestricted use, distribution, and reproduction in any medium, provided the original work is properly cited.

\begin{abstract}
Automated vehicles are designed to free drivers from driving tasks and are expected to improve traffic safety and efficiency when connected via vehicle-to-vehicle communication, that is, connected automated vehicles (CAVs). The time delays and model uncertainties in vehicle control systems pose challenges for automated driving in real world. Ignoring them may render the performance of cooperative driving systems unsatisfactory or even unstable. This paper aims to design a robust and flexible platooning control strategy for CAVs. A centralized control method is presented, where the leader of a CAV platoon collects information from followers, computes the desired accelerations of all controlled vehicles, and broadcasts the desired accelerations to followers. The robust platooning is formulated as a Min-Max Model Predictive Control (MM-MPC) problem, where optimal accelerations are generated to minimize the cost function under the worst case, where the worst case is taken over the possible models. The proposed method is flexible in such a way that it can be applied to both homogeneous platoon and heterogeneous platoon with mixed human-driven and automated controlled vehicles. A third-order linear vehicle model with fixed feedback delay and stochastic actuator lag is used to predict the platoon behavior. Actuator lag is assumed to vary randomly with unknown distributions but a known upper bound. The controller regulates platoon accelerations over a time horizon to minimize a cost function representing driving safety, efficiency, and ride comfort, subject to speed limits, plausible acceleration range, and minimal net spacing. The designed strategy is tested by simulating homogeneous and heterogeneous platoons in a number of typical and extreme scenarios to assess the system stability and performance. The test results demonstrate that the designed control strategy for CAV can ensure the robustness of stability and performance against model uncertainties and feedback delay and outperforms the deterministic MPC based platooning control.
\end{abstract}

\section{Introduction}

Today's traffic systems are facing serious congestion [1]. Automated vehicles using advanced sensing, communication, and control technologies have the potential to increase road capacity and improve traffic operations [2-4]. Adaptive cruise control (ACC) systems, one of the earliest automated vehicle systems, has already entered the market [5-7]. It uses its on-board sensors to detect the ambient environment and regulate the speeds of the vehicle to increase ride comfort. Vehicle-to-vehicle (V2V) and vehicle-to-infrastructure (V2I) communications enable the so-called connected automated vehicles (CAVs), extending the visibility of automated vehicles [8]. When a group of CAVs travel with short intervehicle headway or gap, a platoon is formed [9]. CAVs have more potential to improve traffic performance compared to individual automation, since they can share information and coordinate their behavior to ensure shorter intervehicle distances safely $[2,10,11]$ as demonstrated by field tests $[12$, 13]. With V2I communication between a road side device and electric vehicles, the traffic stability can be improved [14].

Some challenges must be addressed for successful implementation of platooning in real world, including feedback delay, actuator lag, measurement inaccuracy, and heterogeneity in traffic [7, 15-17]. Feedback delay and actuator lag are known to have detrimental effects on string stability $[16,17]$. 
CAVs using platoon leader or predecessor acceleration as feedforward term in control have been shown to be able to compensate delay $[15,18]$. Other delay compensation strategy can be found using predictive control approach [16]. Measurement noise can be treated by using filtering and data fusion techniques [19]. The heterogeneity of traffic can be considered using cooperative control strategies where a joint objective is optimized together within the platoon [20].

Although different methods have been proposed to address the aforementioned challenges, most of them were based on deterministic modelling of vehicle system dynamics and had not been tested systematically against uncertainties. There is no guarantee that existing platooning control systems generate satisfactory performance under real world situations. An adaptive fuzzy sliding-mode control approach is proposed to deal with the model uncertainties, and functional approximation technique is employed to replace the unknown vehicle functions [21]. However, the reliability of using functional approximation technique is not clear. Besides, vehicle speeds can be adjusted through electronic throttle (ET) control. With V2V communication, the opening angle of the ET of the preceding vehicle is available. The following vehicle then adjusts adaptively its ET to avoid collision and follow the speed of the preceding vehicle. Using extended state observer to estimate the change of throttle opening angel and total disturbance, a double-loop integral slidingmode controller for electronic throttle is designed [22]. This controller has robustness to parametric uncertainties of the ET model. An $\mathrm{H}$-infinity control method for a CAV platoon is proposed considering the uncertain vehicle dynamics without considering the feedback delay [23]. The robust control is designed considering the differences of dynamics for CAVs in the platoon. The feasibility of this control method to a heterogenous platoon comprised of CAVs and human-driven vehicle is not given. For a homogeneous platoon, the idea of applying an acceleration of the leader feedforward can also be used to design a robust controller for the following vehicles [1]. The robust control for the CAV platooning control has not been fully explored.

The objective of this paper is to design a robust controller considering the model uncertainties involved in the longitudinal vehicle dynamics. The robust control is designed by considering the parametric uncertainties in the dynamics model of platoons expressed by a third-order linear vehicle model. The robust platooning is then formulated as a MinMax Model Predictive Control (MM-MPC) problem, where optimal desired accelerations are generated to minimize the cost function under the worst case. The controller regulates platoon desired accelerations over a time horizon to minimize the cost function representing driving safety, efficiency, and ride comfort, subject to speed limits, plausible desired acceleration range, and minimal net spacing. The designed control strategy is flexible in such a way that it can be applied to the homogenous platooning control where all the vehicles in the controlled platoon are CAVs and the heterogeneous platooning control where CAVs and human-driven vehicles are mixed.
The paper is organized as follows. We will first introduce longitudinal dynamics models for a CAV, homogenous and heterogenous platoons with CAVs. The proposed robust MM-MPC controllers for a single homogeneous and heterogenous CAV platoon are presented separately. After that, the simulation experiments are designed to verify the performance of the controllers followed by the discussion of the simulation results of the CAV platoons under different control strategies. Finally, we conclude the findings and present future research directions.

\section{Dynamics Models for Longitudinal Behavior of Platoons}

This section presents a longitudinal vehicle dynamics model for state prediction for a single CAV and CAV platoon dynamics model considering actuator lag.

2.1. Single Vehicle Dynamics Model. We introduce a longitudinal dynamics model for a single vehicle $n$, following an exogenous head vehicle, with $x_{n}, v_{n}, a_{n}$, and $l_{n}$ denoting the location, speed, actual acceleration, and vehicle length of the subject vehicle $n$. For a single CAV $n$, the system state $\mathbf{Z}$ is described by the gap error $\Delta s$, relative speed $\Delta v_{n}$ to the preceding vehicle $n-1$, and $a_{n}$; that is, $\mathbf{Z}=\left(\Delta s_{n}, \Delta v_{n}, a_{n}\right)^{T}$, and the control variable is defined as $\mathbf{U}=u_{n}$, where $u_{n}$ is the desired acceleration given to vehicle $n . \Delta s_{n}$ is the deviation between the real gap/net spacing $s_{n}=x_{n-1}-x_{n}-l_{n}$ and the desired gap $s_{n}^{d}$ to vehicle $n-1$; that is, $\Delta s=s_{n}-s_{n}^{d}$. We employ the Constant Time Gap (CTG) policy that is the frequently used by researchers and largely used in the commercial ACC systems to determine the desired gap [11]; that is, $s_{n}^{d}=v_{n} \cdot t^{d}+s_{0}$, where $t^{d}$ is the desired time gap and $s_{0}$ is the minimum gap at standstill. The physical equations for the longitudinal vehicle motion are expressed as (1) when using Newton's second law by modelling the $n$th vehicle as a particle of mass, with $m_{n}$ denoting the mass of vehicle $n$. For simplicity, the time argument is dropped.

$$
\begin{aligned}
m_{n} \cdot \ddot{x}_{n}= & \underbrace{F_{n}}_{\text {tractive force }}-\underbrace{R_{a, n}}_{\text {aerodynamic drag }}-\underbrace{R_{g, n}}_{\text {grade resistance }} \\
& -\underbrace{R_{d, n}}_{\text {mechanical drag }},
\end{aligned}
$$

where $F_{n}$ denotes the driving force produced by the $n$th vehicle's engine which is modelled with a first-order time lag as shown in

$$
\dot{F}_{n}=-\frac{F_{n}}{\tau_{n}^{A}}+\frac{U_{n}}{m_{n} \cdot \tau_{n}^{A}},
$$

where $\tau_{n}^{A}$ denotes the engine time lag for the $n$th vehicle and $U_{n}$ denotes the throttle command input to the $n$th vehicle's engine. By using exact linearization methods, (1) can be linearized as a first-order lag system as expressed by $[16,24$, 25]

$$
\dddot{x}_{n}=\frac{1}{\tau_{n}^{A}} \cdot\left(u_{n}-\ddot{x}_{n}\right) \text {. }
$$


To this end, a third-order model is used to express the longitudinal dynamics model for vehicle $n$ as shown in

$$
\begin{aligned}
\frac{d}{d t} \mathbf{Z} & =\frac{d}{d t}\left(\begin{array}{c}
x_{n-1}-x_{n}-l_{n}-s_{n}^{d} \\
v_{n-1}-v_{n} \\
a_{n}
\end{array}\right) \\
& =\left(\begin{array}{c}
v_{n-1}-v_{n}-a_{n} \cdot t^{d} \\
a_{n-1}-a_{n} \\
\frac{u_{n}-a_{n}}{\tau_{n}^{A}}
\end{array}\right)=\mathbf{f}(\mathbf{Z}, \mathbf{U}, \mathbf{d}),
\end{aligned}
$$

$$
\mathbf{f}(\mathbf{Z}, \mathbf{U}, \mathbf{d})=A \cdot \mathbf{Z}+B \cdot \mathbf{U}+C \cdot \mathbf{d},
$$

where

$$
\begin{aligned}
& A=\left[\begin{array}{ccc}
0 & 1 & -t^{d} \\
0 & 0 & -1 \\
0 & 0 & -\frac{1}{\tau_{n}^{A}}
\end{array}\right] ; \\
& B=\left[\begin{array}{c}
0 \\
0 \\
\frac{1}{\tau_{n}^{A}}
\end{array}\right] ; \\
& C=\left[\begin{array}{l}
0 \\
1 \\
0
\end{array}\right] \\
& \mathbf{d}=a_{n-1} .
\end{aligned}
$$

d denotes the exogeneous disturbance to the vehicle system, which is the actual acceleration of the preceding vehicle $n-1$. If the preceding vehicle is a CAV, the subject vehicle will receive $a_{n-1}$ via $\mathrm{V} 2 \mathrm{~V}$ communication. When using the $\mathrm{V} 2 \mathrm{~V}$ communication to obtain the value of $\mathbf{d}$ is not feasible, the disturbance can be modelled using $a_{n-1}=0$; that is, the vehicle $n-1$ is assumed to travel at the same detected speed in the prediction horizon [16].

2.2. Homogeneous Platoon Dynamics Model. For a homogeneous CAV platoon (e.g., Figure 1(a)) with $N \geq 2$ vehicles, the system state variable is defined as $\mathbf{Z}^{P}=$ $\left(\Delta s_{1}, \Delta v_{1}, a_{1}, \Delta s_{2}, \Delta v_{2}, a_{2}, \ldots, \Delta s_{N}, \Delta v_{N}, a_{N}\right)^{T}$, the command variable is defined as $\mathbf{U}^{P}=\left(u_{1}, u_{2}, \ldots, u_{N}\right)^{T}$, and the disturbance is defined as $\mathbf{d}^{P}=\mathbf{a}_{\mathbf{p}}$, where $\mathbf{a}_{\mathbf{p}}$ is the exogenous head vehicle of the platoon. For each of the vehicles in the homogeneous CAV platoon, the single CAV dynamics model can be applied. Thus the matrix-form system dynamics model for a CAV platoon with $N$ vehicles can be expressed with

$$
\begin{aligned}
& \frac{d}{d t} \mathbf{Z}^{P} \\
& \quad=\frac{d}{d t}\left(\Delta s_{1}, \Delta v_{1}, a_{1}, \Delta s_{2}, \Delta v_{2}, a_{2}, \ldots, \Delta s_{N}, \Delta v_{N}, a_{N}\right)^{T} \\
& \quad=\mathbf{g}\left(\mathbf{Z}^{P}, \mathbf{U}^{P}, \mathbf{d}^{P}\right) \\
& \mathbf{g}\left(\mathbf{Z}^{P}, \mathbf{U}^{P}, \mathbf{d}^{P}\right)=A^{P} \cdot \mathbf{Z}^{P}+B^{P} \cdot \mathbf{U}^{P}+C^{P} \cdot \mathbf{d}^{P}
\end{aligned}
$$

where

$$
\begin{aligned}
& A^{P}=\left[\begin{array}{cccc}
A_{1}^{3 \times 3} & 0^{3 \times 3} & \cdots & 0^{3 \times 3} \\
0^{3 \times 2} & A_{2}^{3 \times 4} & \cdots & 0^{3 \times 3} \\
\vdots & \vdots & \ddots & \vdots \\
0^{3 \times 2} & 0^{3 \times 3} & \cdots & A_{N}^{3 \times 4}
\end{array}\right] ; \\
& B^{P}=\left[\begin{array}{ccc}
B_{1} & & 0 \\
& \ddots & \\
\mathbf{0} & & B_{N}
\end{array}\right] ; \\
& C^{P}=\left[\begin{array}{ll}
C & 0 \\
\mathbf{0} & 0
\end{array}\right]
\end{aligned}
$$$$
A_{1}^{3 \times 3}=\left[\begin{array}{ccc}
0 & 1 & -t^{d} \\
0 & 0 & -1 \\
0 & 0 & -\frac{1}{\tau_{1}^{A}}
\end{array}\right]
$$

$$
A_{i}^{3 \times 4}=\left[\begin{array}{cccc}
0 & 0 & 1 & -t^{d} \\
1 & 0 & 0 & -1 \\
0 & 0 & 0 & -\frac{1}{\tau_{i}^{A}}
\end{array}\right], \quad i=2,3, \ldots, N \text {; }
$$

$$
B_{k}=\left[\begin{array}{c}
0 \\
0 \\
\frac{1}{\tau_{n}^{A}}
\end{array}\right], \quad k=1,2, \ldots, N .
$$

2.3. Heterogeneous Platoon Dynamics Model. When a platoon is comprised of a CAV(s) and a human-driven vehicle(s), a heterogeneous platoon is formed. A heterogenous CAV platoon is given as shown in Figure 1(b). For this platoon, the state and control variables can be defined as $\mathbf{Z}^{H}=\left(\Delta s_{1}, \Delta v_{1}, a_{1}, \Delta s_{2}, \Delta v_{2}, \Delta s_{3}, \Delta v_{3}, a_{3}, \Delta s_{4}, \Delta v_{4}\right)^{T}$ and $\mathbf{U}^{H}=\left(u_{1}, u_{3}\right)^{T}$, and the exogeneous disturbance is $\mathbf{d}^{H}=$ $\left(a_{p}, a_{2}, a_{4}\right)^{T}$. For the human-driven vehicles, the actual accelerations cannot be controlled directly but can be calculated using a car-following model. We use IDM+ to model the collision-free car-following behavior as expressed with (10) and (11), where $\alpha$ is the maximum acceleration, $b$ is the desired 

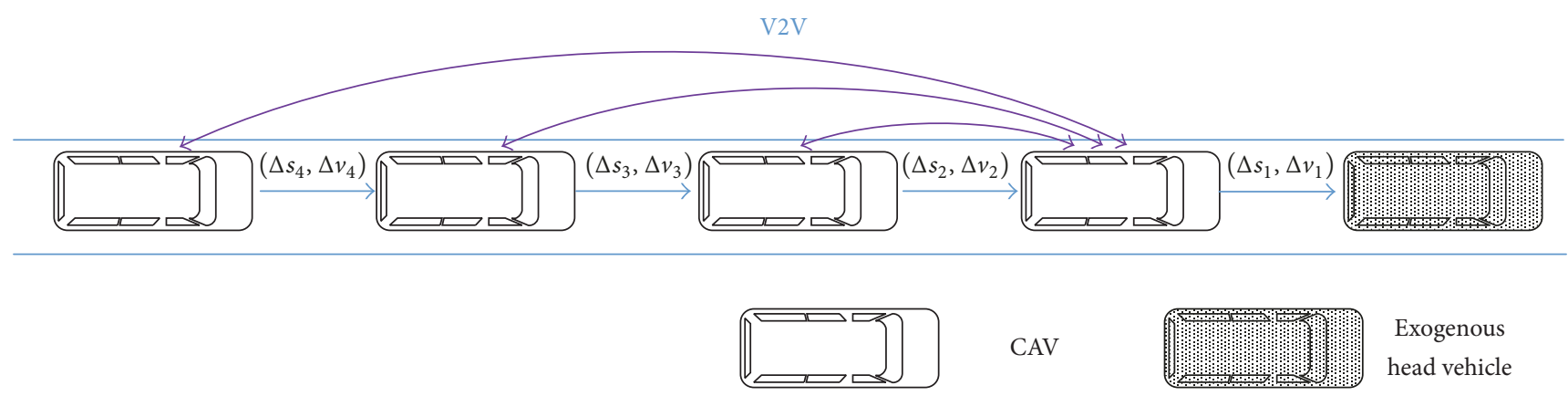

(a) A homogeneous platoon
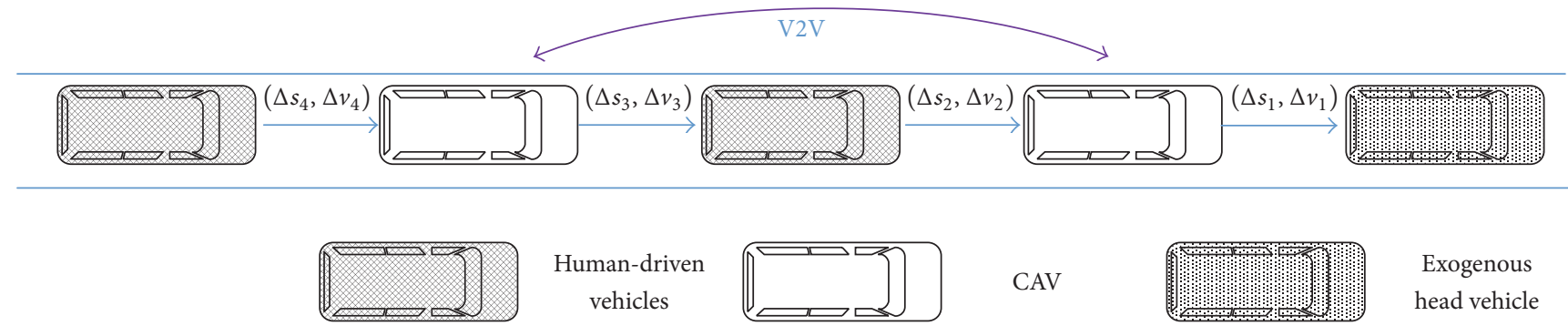

Human-driven

vehicles

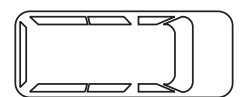

CAV

Exogenous

(b) A heterogenous platoon

FIgURE 1: The platooning formations.

deceleration, and $s^{*}$ is the dynamic desired headway $[26,27]$. To this end, the dynamics model of the heterogenous platoon is as shown in (17) and (18).

$$
\begin{aligned}
& \frac{d x}{d t}=v, \\
& \frac{d v}{d t}=a, \\
& \frac{d v}{d t}=\alpha \cdot \min \left[1-\left(\frac{v}{v_{0}}\right)^{4}, 1-\left(\frac{s^{*}(v, \Delta v)}{s}\right)^{2}\right],
\end{aligned}
$$

where

$$
\begin{aligned}
& s^{*}(v, \Delta v)=\frac{s_{0}+v \cdot T-v \cdot \Delta v}{2 \cdot \sqrt{\alpha \cdot b}}, \\
& \frac{d}{d t} \mathbf{Z}^{H} \\
& \quad=\frac{d}{d t}\left(\Delta s_{1}, \Delta v_{1}, a_{1}, \Delta s_{2}, \Delta v_{2}, \Delta s_{3}, \Delta v_{3}, a_{3}, \Delta s_{4}, \Delta v_{4}\right)^{T} \\
& \quad=\mathbf{h}\left(\mathbf{Z}^{H}, \mathbf{U}^{H}, \mathbf{d}^{H}\right), \\
& \mathbf{h}\left(\mathbf{Z}^{H}, \mathbf{U}^{H}, \mathbf{d}^{H}\right)=A^{H} \cdot \mathbf{Z}^{H}+B^{H} \cdot \mathbf{U}^{H}+C^{H} \cdot \mathbf{d}^{H},
\end{aligned}
$$

where

$$
\begin{aligned}
A^{H} & =\left[\begin{array}{cccc}
A_{n=1} & 0^{3 \times 2} & 0^{3 \times 3} & 0^{3 \times 2} \\
0^{2 \times 2} & D^{2 \times 3} & 0^{2 \times 3} & 0^{2 \times 2} \\
0^{3 \times 2} & 0^{3 \times 3} & A_{n=3} & 0^{3 \times 2} \\
0^{2 \times 2} & 0^{2 \times 3} & 0^{2 \times 2} & D^{2 \times 3}
\end{array}\right] ; \\
B^{H} & =\left[\begin{array}{cc}
0^{2 \times 1} & 0^{2 \times 1} \\
\frac{1}{\tau_{1}^{A}} & 0 \\
0^{4 \times 1} & 0^{4 \times 1} \\
0 & \frac{1}{\tau_{3}^{A}} \\
0^{2 \times 1} & 0^{2 \times 1}
\end{array}\right] ;
\end{aligned}
$$

$$
\begin{aligned}
C^{H} & =\left[\begin{array}{ccc}
C & 0^{3 \times 1} & 0^{3 \times 1} \\
0^{2 \times 1} & E & 0^{2 \times 1} \\
0^{3 \times 1} & C & 0^{3 \times 1} \\
0^{2 \times 1} & 0^{2 \times 1} & E
\end{array}\right] ; \\
D & =\left[\begin{array}{lll}
0 & 0 & 1 \\
1 & 0 & 0
\end{array}\right] ; \\
E & =\left[\begin{array}{c}
-T \\
-1
\end{array}\right] .
\end{aligned}
$$




\section{Design of Robust Controller for Platoon Operation}

In this section, we develop a robust controller to determine the control command by minimizing a running cost function.

3.1. Design Assumptions. The homogeneous platooning controller is designed based on the following assumptions:

(i) The CAVs are homogenous. They have the same vehicle length, desired acceleration boundary, speed limits, and variable actuator lag.

(ii) The accurate feedback information on positions, speeds, and actual accelerations of controlled vehicles can be obtained via on-board sensors and V2V communication, but is subject to a fixed feedback delay $\tau^{S}$. The received state at time $t$ is actually measured at time $t-\tau^{S}$ [16]. As a result of the feedback delay, the initial condition of the system state is not accurate, leading to a model mismatch.

(iii) The controller updates the control command at regular time intervals.

(iv) The CAVs are subjected to the stochastic actuator lag $\tau^{A}(t)$, with $\tau^{A}(t) \in[\alpha, \beta](0<\alpha<\beta)[28,29]$.

\subsection{Platooning Control Formulation}

3.2.1. Min-Max Model Predictive Control Problem. There are several paradigms for robust control of linear systems: $\mathrm{H}_{2}$, $H_{\infty}$, "multi-model" paradigm, and "linear system with a feedback uncertainty" paradigm [30, 31]. For linear systems as in (8), the "multi-model" paradigm works by discovering different linearly independent combination of $\left[\begin{array}{ll}A^{P} & B^{P}\end{array}\right]$. The "linear system with a feedback uncertainty" paradigm can be converted into the form of "multi-model" essentially [30].

The "multi-model" paradigm is chosen considering the stochastic actuator lag $\tau^{A}$. The robust platooning controller is designed by combining Model Predictive Control (MPC) method and the robust control [11]. We formulate the platooning control problem as a Min-Max Model Predictive Control (MM-MPC) problem [30]. We use a centralized control method, where the leader of a CAV platoon collects information from followers, computes the desired accelerations of itself and all followers, and broadcasts the desired accelerations to followers. The designed controller regulates platoon desired accelerations over a time horizon $\left[t_{0}, t_{0}+T_{p}\right]$ to minimize a cost function $J$ representing driving safety, efficiency, and ride comfort. It is formulated as follows:

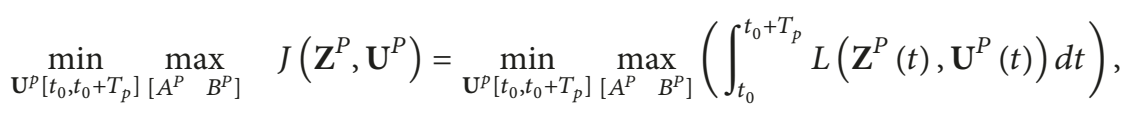

subject to

(1) the system dynamics model of (7),

(2) the initial condition: $\widetilde{\mathbf{Z}}_{t_{0}}^{P}=\mathbf{Z}^{P}\left(t_{0}-\tau^{S}\right)$,

(3) the constraints on states $\mathbf{Z}^{P}(t)$ and $\mathbf{U}^{P}(t)$,

where $L$ denotes the running cost and $\widetilde{\mathbf{Z}}_{t_{0}}^{P}$ represents the initial state for the controlled vehicle system at $t_{0}$.

3.2.2. Cost Specification for Controller. The cost function $L$ is defined as (17), where three different cost terms representing safety, efficiency, and control are included.

$$
L=\underbrace{c_{1} \cdot \sum_{i=1}^{N}\left(\Delta s_{i}\right)^{2}}_{\text {safety }}+\underbrace{c_{2} \cdot \sum_{i=1}^{N}\left(\Delta v_{i}^{2}\right)}_{\text {efficiency }}+\underbrace{c_{3} \cdot \sum_{i=1}^{N}\left(u_{i}\right)^{2}}_{\text {control }} .
$$

The safety cost term implies that the vehicles tend to reach the desired gap. The efficiency cost works by making the following vehicles in the platoon follow the speeds of their preceding vehicles. The control cost penalizes large values of desired acceleration.

3.2.3. Constraints Specification. The constraints on state and control variables are specified as constraints on speeds, gaps, and desired accelerations:
(1) Speed constraint: $v_{i} \in\left[0, v_{\max }\right]$.

(2) Gap constraint: $x_{p}-x_{1}-l_{1} \geq s_{0} ; x_{i}-x_{i+1}-l_{i} \geq s_{0}$.

(3) Acceleration constraint: $u_{i} \in\left[a_{\min }, a_{\max }\right]$.

3.3. Solution Approach. If a nominal model, without considering uncertainties, using a fixed $\left[\begin{array}{ll}A^{P} & B^{P}\end{array}\right]$ is chosen as the dynamics model to design the controller, the controller relaxes to an ordinary MPC problem. We use Sequential Quadratic Programming (SQP) algorithm to generate the optimal control trajectory [32]. When the model uncertainties are considered, we have to solve a MM-MPC problem. The concept of minimizing the worst case is applied to solve the MM-MPC problem, that is, minimizing the largest cost or worst case value of $J$ when a deterministic $\tau^{A}$ is used in the dynamics model to predict the future states of the vehicle systems [25]. To have the largest cost, each possible value of $\tau^{A}$ should be involved. Since there are infinite possibilities of $\tau^{A}$, the computation could be timeconsuming. For simplicity, we discretize the continuous range of the values of $\tau^{A}$ into $M$ intervals of equal size and use the endpoint values to form the models of the dynamics model of the controlled platoon. The solution method of the min-max problem is formulated as an algorithm as shown in Algorithm 1. 
Data: Sensor delay $\tau^{S}$, the boundary of actuator lag $\tau^{A} \in[\alpha, \beta]$, (delayed) system state $\mathbf{Z}^{P}\left(t_{0}-\tau^{S}\right), M$ intervals Results: Optimal control input $u^{*}$ in the horizon $\left[t_{0}, t_{0}+T_{p}\right.$ )

initialization;

for $i \leftarrow 1: M+1$

$\tau_{i}^{A} \leftarrow \alpha+(i-1) \cdot(\beta-\alpha) / M ; u_{i} \leftarrow$ the solution of optimal control problem of Eq. (16) with $\tau^{A} \leftarrow \tau_{i}^{A} ; J_{i} \leftarrow$

the total cost with $u_{i}\left[t_{0}, t_{0}+T_{p}\right]$ using Eq. (16)

end

for $i \leftarrow 2: M+1$

if $J_{1}<J_{i}$

$J_{1} \leftarrow J_{i} ; u_{1}\left[t_{0}, t_{0}+T_{p}\right] \leftarrow u_{i}\left[t_{0}, t_{0}+T_{p}\right]$

end

end

$u^{*}\left[t_{0}, t_{0}+T_{p}\right]=u_{1}\left[t_{0}, t_{0}+T_{p}\right]$

Algorithm 1: The solution for the min-max problem.

\section{Design of Robust Controller for Heterogeneous Platoon Operation}

In this subsection, we first illustrate how to propose a formulation for heterogeneous platooning control like homogeneous platooning control, and then give the detailed design description.

For a platoon of heterogeneous followers like that in Figure 1(b), the CAVs can predict the human-driven vehicle behaviors by using IDM+[20]. Robust heterogeneous platooning control can then be achieved by optimizing a joint cost function the same as (16) and (17) for the whole platoon.

4.1. Design Assumptions. The robust heterogeneous platooning controller is designed under the following additional assumptions compared to homogeneous controller design: (i) The locations and speeds of the human-driven vehicles can be detected by the on-board sensors equipped on the CAVs.

(ii) The controller has imperfect knowledge of the carfollowing behavior. This imperfection is represented by uncertainties in the parameters of IDM+.

4.2. Platooning Control Formulation and Solution. The heterogeneous platooning controller is also formulated as a MinMax Model Predictive Control problem as shown in (18), where the superscript $H$ is used to represent the notations for heterogeneous platooning control. It uses the same format of cost function as the homogeneous platooning controller except that the control cost of the human-driven vehicles is represented by the actual accelerations. The variations of $\tau^{A}$ and the uncertainties in $\alpha$ and $b$ of IDM+ are considered.

$$
\begin{aligned}
& \min _{\mathbf{U}^{H}\left[t_{0}, t_{0}+T_{p}\right]\left[A^{H} B^{H}\right]} \max _{B^{H}} J\left(\mathbf{Z}^{H}, \mathbf{U}^{H}\right)=\min _{\mathbf{U}^{H}\left[t_{0}, t_{0}+T_{p}\right]\left[A^{H} B^{H}\right]} \max _{t_{0}}\left(\int_{t_{0}+T_{p}}^{t^{\prime}} \iota\left(\mathbf{Z}^{H}(t), \mathbf{U}^{H}(t)\right) d t\right), \\
& \iota=\underbrace{c_{1} \cdot \sum_{i=1}^{4}\left(\Delta s_{i}\right)^{2}}_{\text {safety }}+\underbrace{c_{2} \cdot \sum_{i=1}^{4}\left(\Delta v_{i}^{2}\right)}_{\text {efficiency }}+\underbrace{c_{3} \cdot\left(\left(u_{1}\right)^{2}+\left(u_{3}\right)^{2}+\left(a_{2}\right)^{2}+\left(a_{4}\right)^{2}\right)}_{\text {control }},
\end{aligned}
$$

subject to

(1) the system dynamics model of (13) and the dynamics model of human-driven vehicles of (10),

(2) the initial condition and the constraints: they are the same as that described in the homogenous platooning controller design.

The Min-Max Model Predictive Control problem for the heterogeneous includes the variation of $\tau^{A}$ and the uncertainties in $\alpha$ and $b$ of IDM+. The solution for this problem is essentially the same as that used in the homogeneous platooning controller design. We discretize the continuous range of the values of $\tau^{A}$ with the same method as that used in the homogeneous platooning controller design. After that, the problem is solved as for the homogeneous platooning controller design.

\section{Simulation Experimental Design}

This section presents the experimental design to assess the performance of the designed platooning controllers, including selected simulation scenarios, controller parameter settings, and the performance indicators. 
TABLE 1: Simulation scenarios for a homogeneous platooning control test.

\begin{tabular}{lc}
\hline$\tau^{A}(s)$ & Control strategy \\
\hline$[0.2,0.8]$ & Nominal MPC $\left(\tau^{A}=0.2\right)$ \\
{$[0.2,0.8]$} & Robust MM-MPC $\left(\tau^{A}(t) \in[0.2,0.8]\right)$ \\
{$[0.8,0.9]$} & Nominal MPC $\left(\tau^{A}=0.2\right)$ \\
{$[0.8,0.9]$} & Robust MM-MPC $\left(\tau^{A}(t) \in[0.2,0.8]\right)$ \\
\hline
\end{tabular}

5.1. Simulation Scenarios. To test the robustness of the robust MM-MPC controller for a homogeneous CAV platoon and the flexibility of the controller to a heterogeneous platoon control, different simulation scenarios are used. Generally $\tau^{S}$ is between 0.1 and $0.3 \mathrm{~s}$ [16]. We choose $\tau^{S}=0.2 \mathrm{~s}$. $\tau^{A}(t) \in[0.2,0.8] \mathrm{s}$ is chosen as designed bound of $\tau^{A}$. We compare the designed robust controller with a deterministic nominal MPC controller using a fixed $\tau^{A}=0.2 \mathrm{~s}$. The two controllers are compared under the designed scenario with $\tau^{A}(t) \in[0.2,0.8] \mathrm{s}$ and unplanned scenario with $\tau^{A}(t) \in$ $[0.8,0.9] \mathrm{s}$; that is, the actuator lag of vehicle systems is outside the assumed bounds [30]. The selected simulation scenarios are as shown in Table 1. The heterogeneous platooning controller is tested with imperfect knowledge of the car-following behavior of human-driven vehicles by using different parameters of IDM+ used by human-driven vehicles in the platoon. The selected scenarios are as shown in Table 2.

5.2. Parameter Settings. We choose $N=4$ to demonstrate platoon control, since it can sufficiently show the performances of the controlled platoon as shown in Figures $1(\mathrm{a})$ and $1(\mathrm{~b})$ [13]. The parameter setting for CAVs in the homogenous and heterogeneous platooning controllers is the same. The platoons follow an exogenous head vehicle that has a designated speed profile. The total simulation time is 50 seconds (s). To clearly show the performances of the controllers, we use a step function of acceleration for the exogenous head vehicle. It starts with an initial speed of $25 \mathrm{~m} / \mathrm{s}$ and decelerates with $-4 \mathrm{~m} / \mathrm{s}^{2}$ from 3 to $5 \mathrm{~s}$ and then accelerates with $1 \mathrm{~m} / \mathrm{s}^{2}$ from $27 \mathrm{~s}$ to $35 \mathrm{~s}$. For other time slots, its acceleration is $0 \mathrm{~m} / \mathrm{s}^{2}$. For the controllers, the parameters are set as follows: $t^{d}=1 \mathrm{~s}$ [33], $s_{0}=2 \mathrm{~m}, T_{p}=5 \mathrm{~s}, c_{1}=0.6$, $c_{2}=0.5, c_{3}=0.6, a_{\max }=1.5 \mathrm{~m} / \mathrm{s}^{2}, a_{\min }=-8 \mathrm{~m} / \mathrm{s}^{2}, l_{i}=4 \mathrm{~m}$ ( $i=1,2,3,4), M=19$, and $v_{\max }=120 / 3.6 \mathrm{~m} / \mathrm{s}$.

The controller parameters have been manually tuned that the controller tracks the exogenous head vehicle responsively and has small overshoots and no oscillations in the case where there is a fixed sensor delay $\tau^{S}=0.2 \mathrm{~s}$ but no actuator lag. In the same case, the time horizon $T_{p}$ is chosen by preliminary simulations. The total running cost barely changes with higher values of $T_{p}$. Systematic tuning methods of MPC can be found in [34].

For the heterogeneous platooning control, we assume the controller has imperfect knowledge of the human-driven vehicles. The controller assumes that the parameters of the IDM+ are $\alpha=1.25 \mathrm{~m} / \mathrm{s}^{2}, b=2.09 \mathrm{~m} / \mathrm{s}^{2}$, and $T=1.2 \mathrm{~s}$, while actually the parameters of the IDM+ are $\alpha=1.1 \mathrm{~m} / \mathrm{s}^{2}$, $b=2 \mathrm{~m} / \mathrm{s}^{2}$, and $T=1.2 \mathrm{~s}$. Both simulation time step and controller sampling step are $0.2 \mathrm{~s}$. The simulation starts with equilibrium conditions for each CAV and human-driven vehicle.

5.3. Performance Assessment Indicators. Several indicators are selected to assess the performance of the CAV platoon under different control strategies: (1) the accumulation of running cost reflecting the control objectives of the controller; (2) costs of the first controlled vehicle (1st vehicle) and the last vehicle (4th vehicle); (3) maximum absolute actual acceleration, relative speed, and gap error for the first and last vehicle; (4) string stability.

\section{Simulation Results and Discussion}

In this section, the simulation results are shown and analyzed and the discussion is presented thereafter.

6.1. Homogeneous Platooning Control Performance. The simulations are performed separately with the nominal MPC controller (deterministic controller with actuator lag $\tau^{A}=$ $0.2 \mathrm{~s}$ ) and the robust MM-MPC controller. The performances are shown with figures of the variation of actual acceleration, relative speed, and gap error as shown in Figure 2 and those of assessment indicators are shown in second and third columns in Table 3. As depicted in Figure 2, the nominal MPC and the designed robust MM-MPC controller generate reasonable behavior when $\tau^{A}(t) \in[0.2,0.8] \mathrm{s}$. When the exogenous head vehicle decelerates, the relative speed and gap error of the first vehicle become negative while other vehicles are still in the equilibrium states, and the cost of the controller starts to increase. Both controllers work by reducing the costs and give control commands to reduce the relative speeds and gap errors caused by the deceleration of the exogenous head vehicle. The first vehicle then starts to decelerate. After that, the second vehicle's relative speed and gap error become negative as well and costs are generated, and the controller has to reduce the costs caused by the relative speeds and gap errors of the first and second vehicles while considering the control costs. It can easily be observed in Figure 2 that the changes of actual accelerations start from the first vehicle to the last vehicle sequentially.

The behavior of the first vehicle is more sensitive to the behavior of the exogenous head vehicle, and this can be reflected in Figure 2 and values of costs in Table 3. Both controllers can settle to new equilibrium where the cost is zero after certain time, and the maximum of absolute actual acceleration, relative speed, and gap error of the 4 th vehicle is smaller than that of the first vehicle. To this end, they can ensure string stability.

6.2. Robustness of MM-MPC Controller. When it comes to the scenario with $\tau^{A}(t) \in[0.8,0.9] \mathrm{s}$, the performances of the nominal MPC controller (deterministic controller with actuator lag $\tau^{A}=0.2 \mathrm{~s}$ ) and the designed robust MM-MPC controller differ as shown in Figure 3 and the assessment indicators are shown in the fourth and fifth columns in Table 3. The nominal MPC controller can plausibly reach 
TABLE 2: Simulation scenarios for a heterogeneous platooning control test.

\begin{tabular}{lccc}
\hline$\tau^{A}(\mathrm{~s})$ & $\alpha\left(\mathrm{m} / \mathrm{s}^{2}\right)$ & $b\left(\mathrm{~m} / \mathrm{s}^{2}\right)$ & Control strategy \\
\hline$[0.2,0.8]$ & 1.1 & 2 & Nominal MPC $\left(\tau^{A}=0.2, \alpha=1.25, b=2.09\right)$ \\
{$[0.2,0.8]$} & 1.1 & 2 & Robust MM-MPC $\left(\tau^{A}(t) \in[0.2,0.8], \alpha=1.25, b=2.09\right)$ \\
{$[0.8,0.9]$} & 1.1 & 2 & Nominal MPC $\left(\tau^{A}=0.2, \alpha=1.25, b=2.09\right)$ \\
{$[0.8,0.9]$} & 1.1 & 2 & Robust MM-MPC $\left(\tau^{A}(t) \in[0.2,0.8], \alpha=1.25, b=2.09\right)$ \\
\hline
\end{tabular}

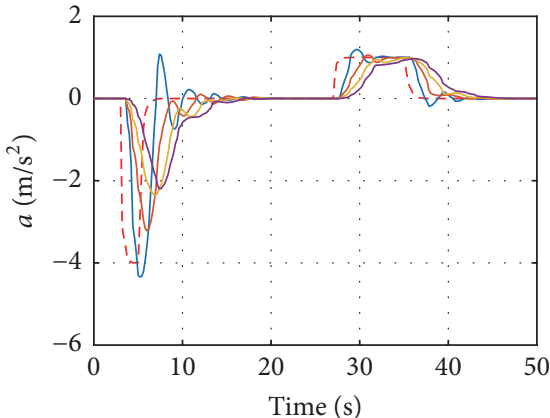

- - Exogenous head vehicle

- 1st

- 2nd

3rd

- 4 th

(a) Nominal MPC actual acceleration

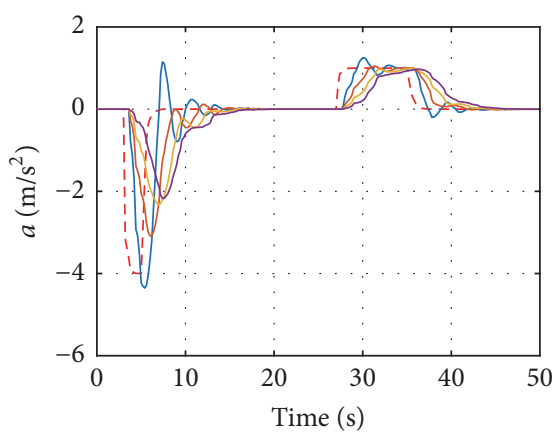

- - - Exogenous head vehicle

- 1st

- 2nd

3rd

- 4 th

(d) MM-MPC actual acceleration
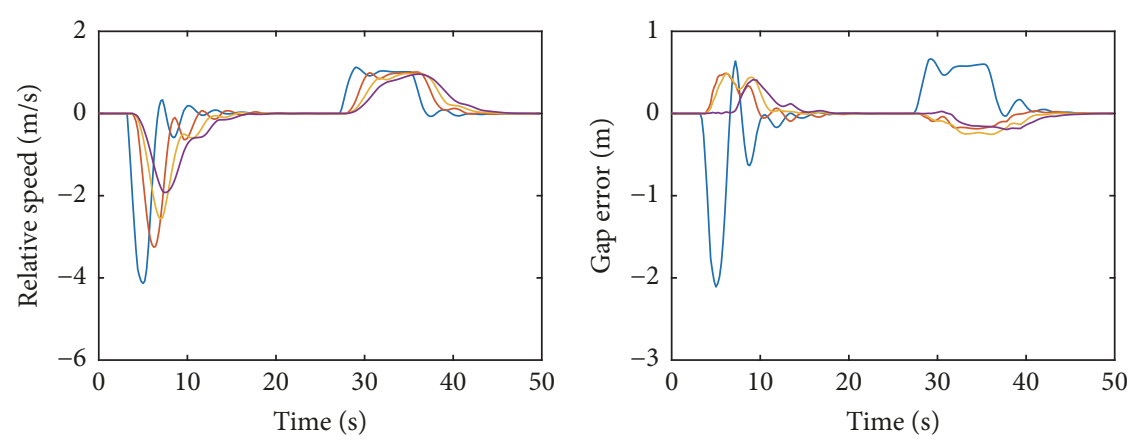

$\begin{array}{ll}-1 \text { st } & - \text { 3rd } \\ \text { 2nd } & \text { - }\end{array}$

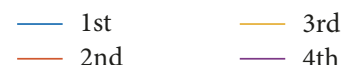

(b) Nominal MPC relative speed

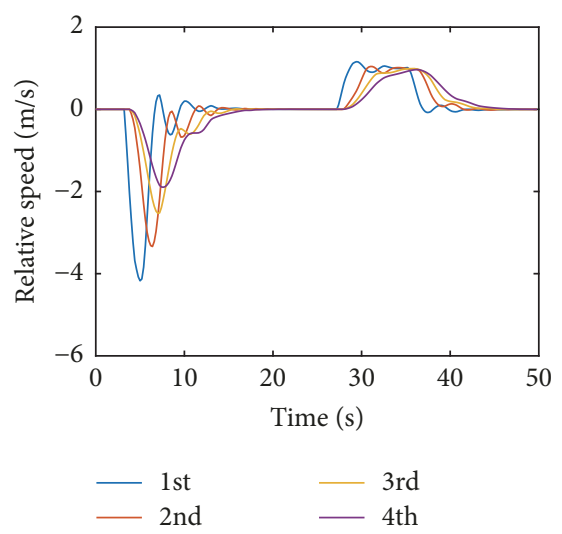

(e) MM-MPC relative speed (c) Nominal MPC gap error

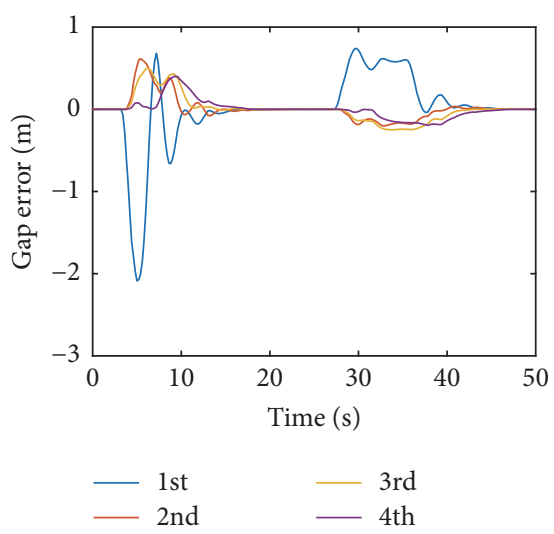

(f) MM-MPC gap error

FIGURE 2: Comparison of nominal MPC $\left(\tau^{A}=0.2 \mathrm{~s}\right)$ and MM-MPC (designed with $\left.\tau^{A}(t) \in[0.2,0.8] \mathrm{s}\right)$ for homogenous platooning control while actual $\tau^{A}(t) \in[0.2,0.8] \mathrm{s}$.

a new equilibrium state; however, that cannot be achieved within the simulation time as shown in Figures 3(a), 3(b), and 3(c). There exist many oscillations, but the nominal MPC controller may ensure platoon stability since the maximum of absolute actual acceleration, relative speed, and gap error of the 4th vehicle is smaller than that of the first vehicle as shown in Table 3. By comparison, the robust MM-MPC controller can still settle to new equilibrium and thus can ensure local and string stability. Besides, the total cost of the platoon using MM-MPC controller is $26.38 \%$ lower than that using the nominal MPC controller. This illustrates that the MM-MPC controller is quite robust against large model uncertainties. As opposed to the robust MM-MPC controller, the nominal MPC controller cannot handle large model uncertainties.

6.3. Heterogeneous Platooning Control Performance. The nominal MPC controller and the designed robust MM-MPC controller for the heterogeneous platoon are tested with imperfect knowledge of the car-following model IDM+. The simulations are conducted with different parameter settings of the IDM+ used in the controllers and the human-driven vehicles. The performances of the nominal MPC controller 


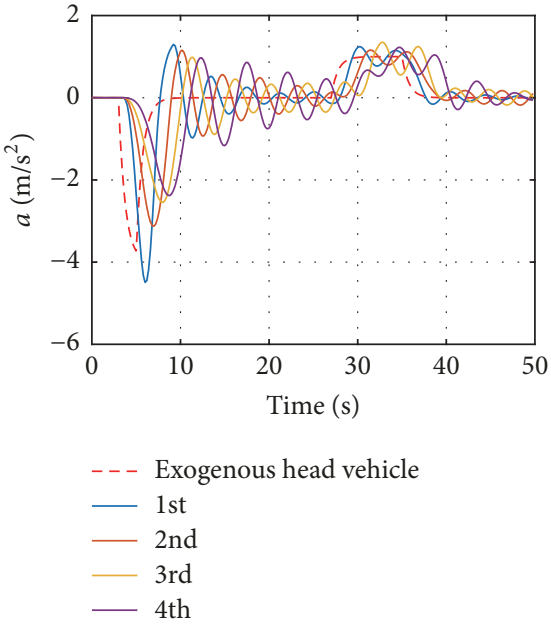

(a) Nominal MPC actual acceleration

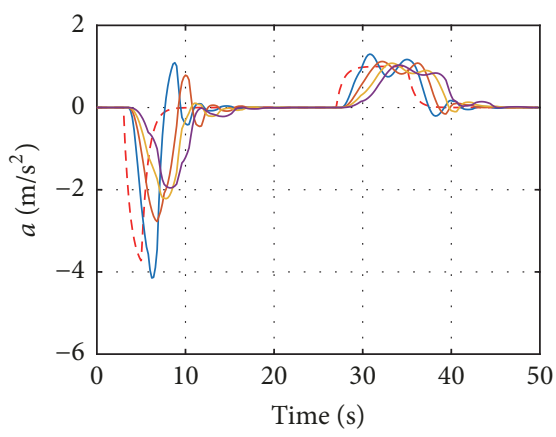

- - Exogenous head vehicle

- 1 st

— 2nd

3rd

- 4th

(d) MM-MPC actual acceleration

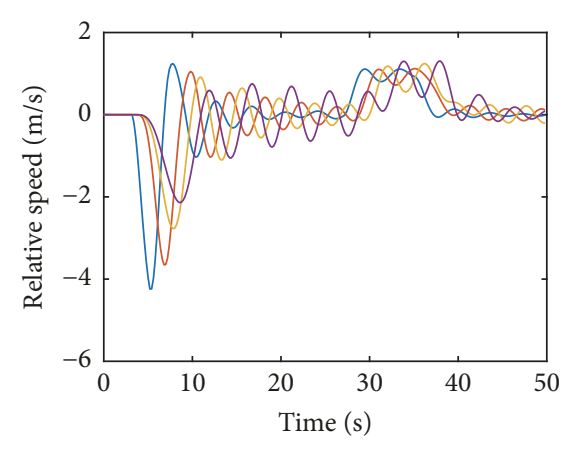

$\begin{array}{ll}\text { 1st } & \text { 3rd } \\ \text { 2nd } & \text { - }\end{array}$

(b) Nominal MPC relative speed

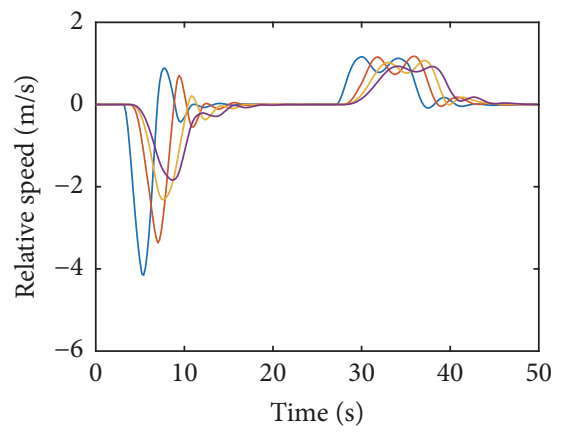

$\begin{array}{ll}\text { 1st } & \text { 3rd } \\ - & \text { 2nd }\end{array}$

(e) MM-MPC relative speed

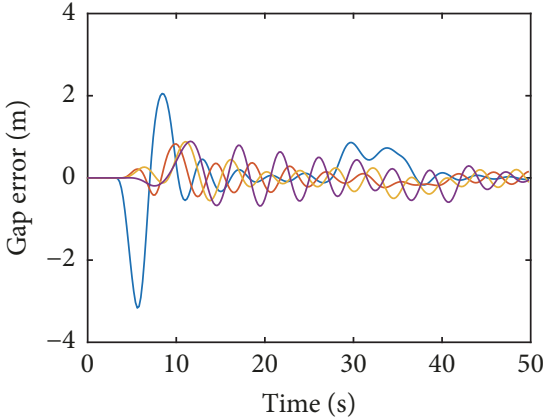

- 1st $\quad$ 3rd

- 2nd - 4th

(c) Nominal MPC gap error

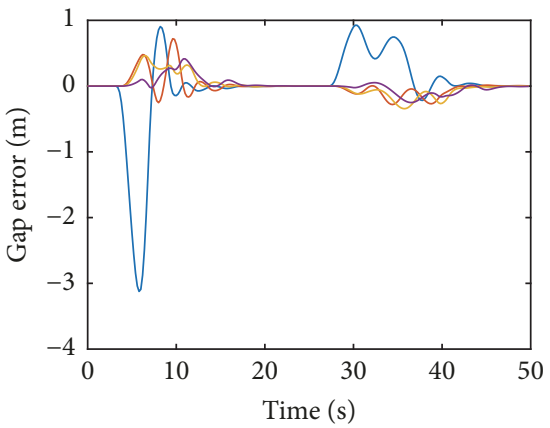

- 1st $\quad$ - 3rd

Figure 3: Comparison of nominal MPC $\left(\tau^{A}=0.2 \mathrm{~s}\right)$ and MM-MPC (designed with $\left.\tau^{A}(t) \in[0.2,0.8] \mathrm{s}\right)$ for homogenous platooning control while actual $\tau^{A}(t) \in[0.8,0.9] \mathrm{s}$.

TABLE 3: Performance results: homogeneous platooning controllers.

\begin{tabular}{|c|c|c|c|c|}
\hline \multirow{2}{*}{ Indicators } & Nominal MPC & MM-MPC & Nominal MPC & MM-MPC \\
\hline & \multicolumn{2}{|c|}{$\tau^{S}=0.2 \mathrm{~s}, \tau^{A}(t) \in[0.2,0.8] \mathrm{s}$} & \multicolumn{2}{|c|}{$\tau^{S}=0.2 \mathrm{~s}, \tau^{A}(t) \in[0.8,0.9] \mathrm{s}$} \\
\hline$\sum_{i=1}^{4} J_{i}$ & 617,57 & 615,19 & 936,75 & 689,59 \\
\hline$J_{1}$ & 248,43 & 248,65 & 352,58 & 308,49 \\
\hline$J_{4}$ & 99,99 & 99,01 & 187,37 & 98,39 \\
\hline $\max \left|\Delta v_{1}^{+}\right|(\mathrm{m} / \mathrm{s})$ & 1,13 & 1,16 & 1,24 & 1,16 \\
\hline $\max \left|\Delta v_{4}^{+}\right|(\mathrm{m} / \mathrm{s})$ & 0,96 & 0,97 & 1,30 & 0,93 \\
\hline $\max \left|a_{1}^{+}\right|\left(\mathrm{m} / \mathrm{s}^{2}\right)$ & 1,19 & 1,25 & 1,30 & 1,30 \\
\hline $\max \left|a_{4}^{+}\right|\left(\mathrm{m} / \mathrm{s}^{2}\right)$ & 0,97 & 0,97 & 1,23 & 1,03 \\
\hline $\max \left|\Delta s_{1}^{+}\right|(\mathrm{m})$ & 0,66 & 0,74 & 2,05 & 0,93 \\
\hline $\max \left|\Delta s_{4}^{+}\right|(\mathrm{m})$ & 0,41 & 0,40 & 0,89 & 0,41 \\
\hline $\max \left|\Delta v_{1}^{-}\right|(\mathrm{m} / \mathrm{s})$ & 4,13 & 4,17 & 4,24 & 4,15 \\
\hline $\max \left|\Delta v_{4}^{-}\right|(\mathrm{m} / \mathrm{s})$ & 1,92 & 1,90 & 2,14 & 1,84 \\
\hline $\max \left|a_{1}^{-}\right|\left(\mathrm{m} / \mathrm{s}^{2}\right)$ & 4,34 & 4,35 & 4,49 & 4,15 \\
\hline $\max \left|a_{4}^{-}\right|\left(\mathrm{m} / \mathrm{s}^{2}\right)$ & 2,20 & 2,17 & 2,38 & 1,95 \\
\hline $\max \left|\Delta s_{1}^{-}\right|(\mathrm{m})$ & 2,11 & 2,09 & 3,17 & 3,12 \\
\hline $\max \left|\Delta s_{4}^{-}\right|(\mathrm{m})$ & 0,20 & 0,19 & 0,68 & 0,25 \\
\hline
\end{tabular}




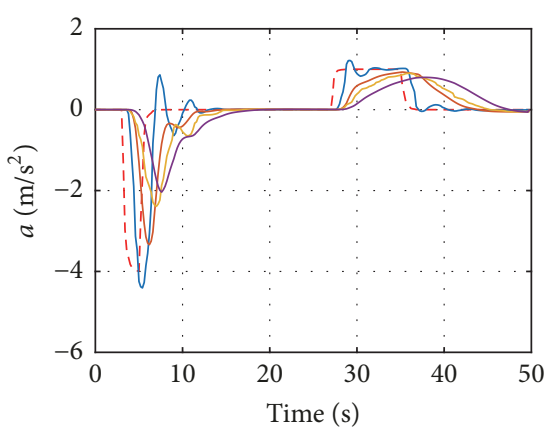

- - - Exogenous head vehicle

- 1 st

- 2nd

3rd

- 4 th

(a) Nominal MPC actual acceleration

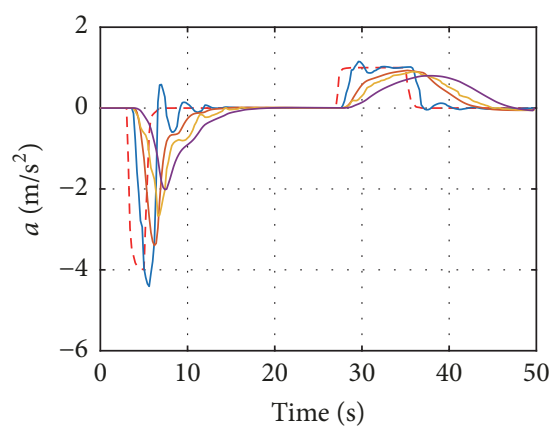

- - Exogenous head vehicle

- 1 st

- 2nd

3rd

4th

(d) MM-MPC actual acceleration

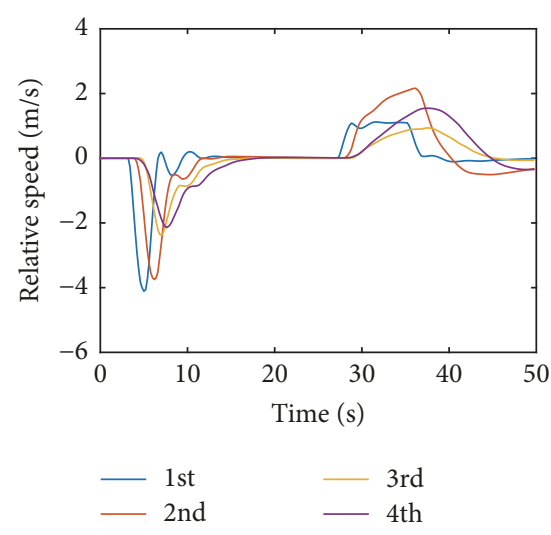

(b) Nominal MPC relative speed

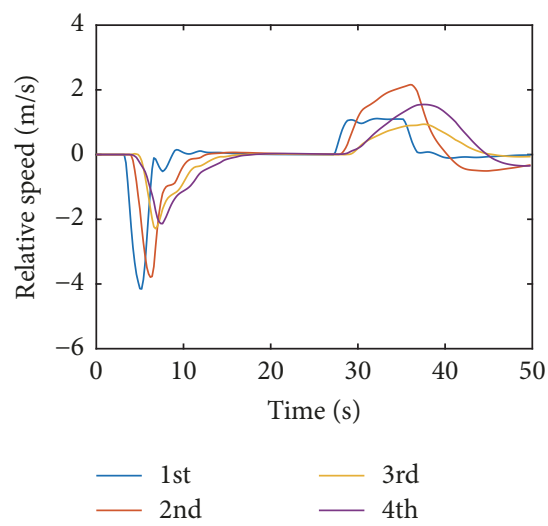

(e) MM-MPC relative speed
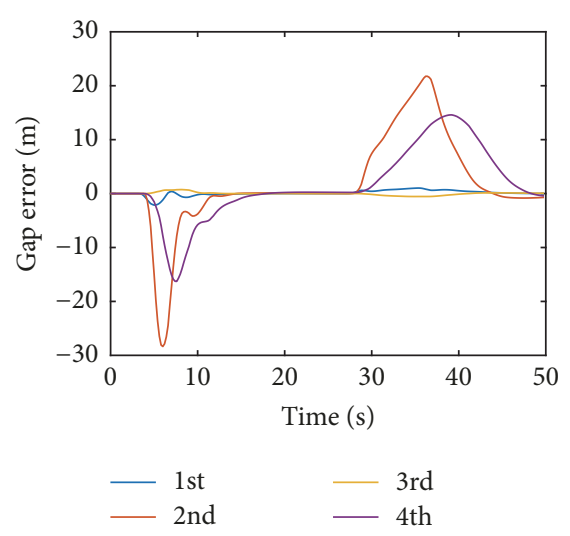

(c) Nominal MPC gap error

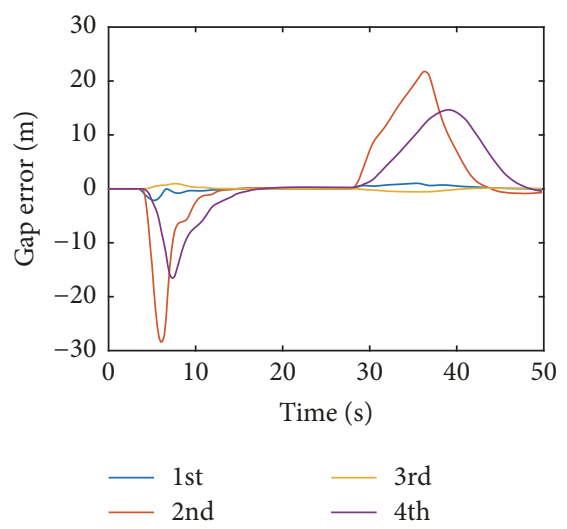

(f) MM-MPC gap error

FIGURE 4: Comparison of nominal MPC $\left(\tau^{A}=0.2 \mathrm{~s}\right)$ and MM-MPC (designed with $\left.\tau^{A}(t) \in[0.2,0.8] \mathrm{s}\right)$ for heterogeneous platooning control while actual $\tau^{A}(t) \in[0.2,0.8] \mathrm{s}$.

(deterministic controller with actuator lag $\tau^{A}=0.2 \mathrm{~s}$ ) and the designed robust MM-MPC controller are shown in Figures 4 and 5 and Table 4.

When $\tau^{A}(t) \in[0.2,0.8] \mathrm{s}$, the performances of nominal MPC controller and the designed robust MM-MPC controller are similar. They both generate reasonable behavior as analyzed in homogeneous platooning control performance and settle to the new equilibrium state within the simulation time. However, when it comes to $\tau^{A}(t) \in[0.8,0.9] \mathrm{s}$, the nominal MPC controller has more oscillations before reaching the new equilibrium state. The ability to reach a new equilibrium state and keep string stability is plausible for the nominal MPC controller while being obvious for the MMMPC controller. Besides, MM-MPC controller brings $12.55 \%$ total platoon cost reduction compared with the nominal MPC controller.

The simulation results confirm the feasibility and robustness of the MM-MPC controller to homogenous platooning control and flexibility to heterogenous platooning control.
6.4. Discussion. For the nominal MPC controller, the actuator lag $\tau^{A}=0.2 \mathrm{~s}$ is chosen for open-loop optimal control. The selection is based on the preliminary simulations. If $\tau^{A}=0.5 \mathrm{~s}$ is chosen for open-loop optimal control, the variation of the actual accelerations has more oscillations and the total cost is higher than using $\tau^{A}=0.2 \mathrm{~s}$ for open-loop optimal control and the MM-MPC controller in the case where the actual actuator lag is a fixed value of $0.2 \mathrm{~s}$. This may be caused by the higher absolute values of desired acceleration when $\tau^{A}=0.5 \mathrm{~s}$ is chosen for open-loop optimal control, since it uses a larger value of actuator lag as model parameter.

The solution method for MM-MPC is clear and obvious enough; however, the relation of $M$ and the length of the bound of $\tau^{A}(t)$ is not deeply explored. It is noticable that the larger $M$ is, the longer the computation time will be. However, with a larger $M$, the performance of the robustness of the controller may be better as the worst case situation is close to that in the real world. There is a tradeoff between the computation time and the performance when choosing the 

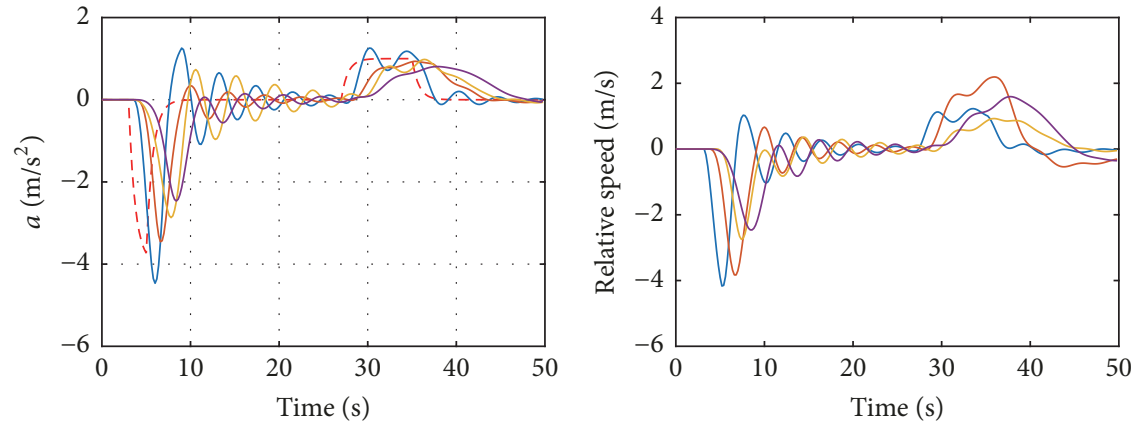

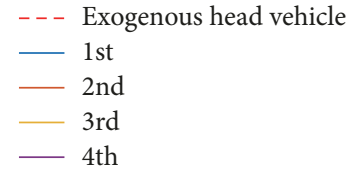

(a) Nominal MPC actual acceleration

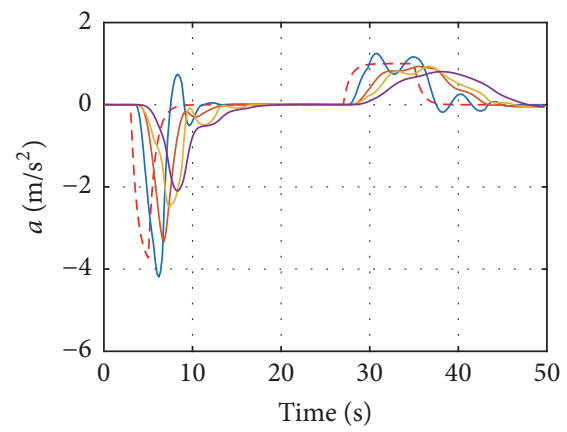

- - - Exogenous head vehicle

- 1 st

- 2nd

- 3rd

- 4th

(d) MM-MPC actual acceleration

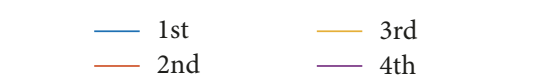

(b) Nominal MPC relative speed

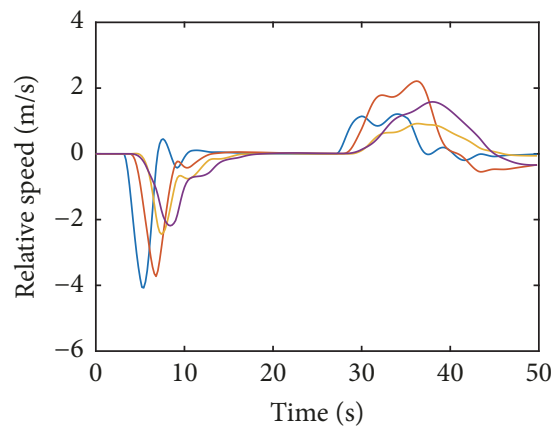

$\begin{array}{ll}\text { - 1st } & -3 \mathrm{rd} \\ \text { 2nd } & \text { - }\end{array}$

(e) MM-MPC relative speed

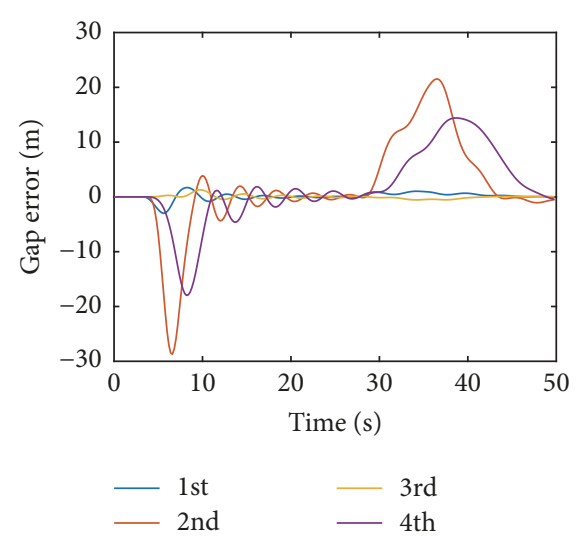

(c) Nominal MPC gap error

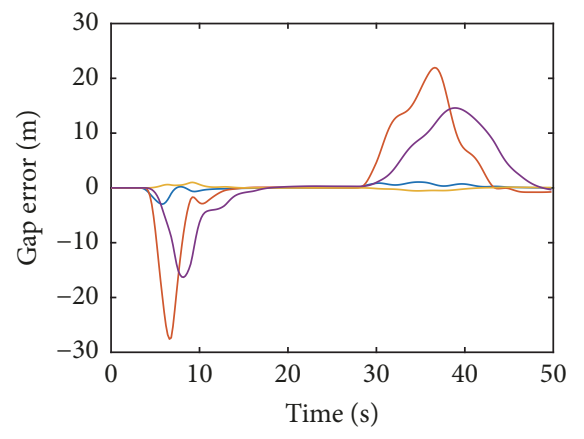

$\begin{array}{ll}\text { 1st } & \text { 3rd } \\ \text { 2nd } & \text { rth }\end{array}$

(f) MM-MPC gap error

FIGURE 5: Comparison of nominal MPC $\left(\tau^{A}=0.2 \mathrm{~s}\right)$ and MM-MPC (designed with $\left.\tau^{A}(t) \in[0.2,0.8] \mathrm{s}\right)$ for heterogeneous platooning control while actual $\tau^{A}(t) \in[0.8,0.9] \mathrm{s}$.

TABLE 4: Performance results: heterogeneous platooning controllers.

\begin{tabular}{|c|c|c|c|c|}
\hline \multirow{2}{*}{ Indicators } & Nominal MPC & MM-MPC & Nominal MPC & MM-MPC \\
\hline & \multicolumn{2}{|c|}{$\tau^{S}=0.2 \mathrm{~s}, \tau^{A}(t) \in[0.2,0.8] \mathrm{s}$} & \multicolumn{2}{|c|}{$\tau^{S}=0.2 \mathrm{~s}, \tau^{A}(t) \in[0.8,0.9] \mathrm{s}$} \\
\hline$\sum_{i=1}^{4} J_{i}$ & 938,74 & 933,82 & 1120,52 & 988,19 \\
\hline$J_{1}$ & 249,38 & 250,08 & 348,66 & 301,35 \\
\hline$J_{4}$ & 212,97 & 211,75 & 229,23 & 209,92 \\
\hline $\max \left|\Delta v_{1}^{+}\right|(\mathrm{m} / \mathrm{s})$ & 1,12 & 1,11 & 1,23 & 1,21 \\
\hline $\max \left|\Delta v_{4}^{+}\right|(\mathrm{m} / \mathrm{s})$ & 1,55 & 1,55 & 1,59 & 1,58 \\
\hline $\max \left|a_{1}^{+}\right|\left(\mathrm{m} / \mathrm{s}^{2}\right)$ & 1,22 & 1,15 & 1,26 & 1,25 \\
\hline $\max \left|a_{4}^{+}\right|\left(\mathrm{m} / \mathrm{s}^{2}\right)$ & 0,80 & 0,80 & 0,81 & 0,81 \\
\hline $\max \left|\Delta s_{1}^{+}\right|(\mathrm{m})$ & 1,02 & 1,03 & 1,71 & 1,07 \\
\hline $\max \left|\Delta s_{4}^{+}\right|(\mathrm{m})$ & 14,59 & 14,65 & 14,41 & 14,59 \\
\hline $\max \left|\Delta v_{1}^{-}\right|(\mathrm{m} / \mathrm{s})$ & 4,11 & 4,15 & 4,17 & 4,08 \\
\hline $\max \left|\Delta v_{4}^{-}\right|(\mathrm{m} / \mathrm{s})$ & 2,14 & 2,14 & 2,46 & 2,19 \\
\hline $\max \left|a_{1}^{-}\right|\left(\mathrm{m} / \mathrm{s}^{2}\right)$ & 4,41 & 4,41 & 4,47 & 4,19 \\
\hline $\max \left|a_{4}^{-}\right|\left(\mathrm{m} / \mathrm{s}^{2}\right)$ & 2,04 & 2,01 & 2,46 & 2,09 \\
\hline $\max \left|\Delta s_{1}^{-}\right|(\mathrm{m})$ & 2,14 & 2,14 & 2,99 & 2,96 \\
\hline $\max \left|\Delta s_{4}^{-}\right|(\mathrm{m})$ & 16,28 & 16,55 & 17,97 & 16,31 \\
\hline
\end{tabular}


value of $M$. The solution method can be replaced by using linear matrix inequalities [30].

Even though the controllers have imperfect knowledge of the car-following model, the good performance of the controller can be kept. This implies the controllers are not sensitive to the parameters of the car-following model, and the controllers for homogeneous platooning control may be converted for heterogeneous platooning control easily. It is noticable that the positive benefits of using MM-MPC controller are larger with homogeneous platooning than with the heterogeneous platooning. This may be explained by the fact that the controller cannot give command to the human-driven vehicle directly. The simulation results show the feasibility and robustness of the MM-MPC controller to homogeneous platooning control and flexibility to heterogeneous platooning control.

The simulation results indicate that, with model uncertainties, the robust control is needed for guaranteeing the benefits of the designed controller. This paper focuses on using the parameter uncertainties to represent the model uncertainties. However, we have not explored other types of methods of representing model uncertainties. To ensure the robustness of the proposed controller, it is suggested to have insights into the possible model uncertainties in the real traffic environment and test the controllers before putting the automated vehicles with the designed controllers into production.

\section{Conclusion}

This paper proposed a robust MM-MPC controller for vehicle homogeneous and heterogeneous platooning control, taking into account the feedback delay and model parametric uncertainties. Unlike the deterministic MPC, the robust MMMPC controller is formulated as a min-max problem and generates the desired acceleration by selecting the solution of the worst case. The solution algorithm entails discretizing the continuous range of the uncertain parameter range into finite intervals and using boundaries of each grid to seek the worst case. Several simulations are conducted to demonstrate the robustness and flexibility of the proposed MM-MPC controller with reference to the nominal deterministic MPC controller under and outside the designed parameter range, respectively. In all situations, the robust MM-MPC controller outperforms the nominal MPC controller and the benefits of the robust MM-MPC are much more pronounced where the uncertainties are outside the designed bounds.

Application of the MM-MPC controller in heterogeneous platoon control validates the flexibility of the proposed control approach and further demonstrates the robustness of the controller against uncertainties in mixed traffic. The proposed controller has the potential to improve traffic operations, in particular traffic stability, due to its robust performance against uncertainties and system delays.

This research will be extended to robust control design under stochastic feedback delay and input uncertainties in addition to model parametric uncertainties. Future research is also directed towards the analytic approach using Lyapunov theory to guarantee string stability of vehicle platoons and robust lane change control in mixed traffic to improve traffic operations.

\section{Disclosure}

An earlier version of this work was presented at the 97th Transportation Research Board (TRB) Annual Meeting in 2018.

\section{Conflicts of Interest}

The authors declare that they have no conflicts of interest.

\section{Acknowledgments}

This research is sponsored by Rijkswaterstaat and the China Scholarship Council.

\section{References}

[1] A. Ghasemi, R. Kazemi, and S. Azadi, "Exact stability of a platoon of vehicles by considering time delay and lag," Journal of Mechanical Science and Technology, vol. 29, no. 2, pp. 799-805, 2015.

[2] B. Van Arem, C. J. G. Van Driel, and R. Visser, "The impact of cooperative adaptive cruise control on traffic-flow characteristics," IEEE Transactions on Intelligent Transportation Systems, vol. 7, no. 4, pp. 429-436, 2006.

[3] K. C. Dey, L. Yan, X. Wang et al., "A Review of Communication, Driver Characteristics, and Controls Aspects of Cooperative Adaptive Cruise Control (CACC)," IEEE Transactions on Intelligent Transportation Systems, vol. 17, no. 2, pp. 491-509, 2016.

[4] M. Wang, W. Daamen, S. P. Hoogendoorn, and B. Van Arem, "Cooperative Car-Following Control: Distributed Algorithm and Impact on Moving Jam Features," IEEE Transactions on Intelligent Transportation Systems, vol. 17, no. 5, pp. 1459-1471, 2016.

[5] J. VanderWerf, S. Shladover, N. Kourjanskaia, M. Miller, and H. Krishnan, "Modeling effects of driver control assistance systems on traffic," Transportation Research Record, no. 1748, pp. 167-174, 2001.

[6] Q. Xu and R. Sengupta, "Simulation, analysis, and comparison of ACC and CACC in highway merging control," in Proceedings of the 2003 IEEE Intelligent Vehicles Symposium, IV 2003, pp. 237-242, USA, June 2003.

[7] G. J. L. Naus, R. P. A. Vugts, J. Ploeg, M. J. G. van de Molengraft, and M. Steinbuch, "String-stable CACC design and experimental validation: a frequency-domain approach," IEEE Transactions on Vehicular Technology, vol. 59, no. 9, pp. 42684279, 2010.

[8] J. Rios-Torres and A. A. Malikopoulos, "A Survey on the Coordination of Connected and Automated Vehicles at Intersections and Merging at Highway On-Ramps," IEEE Transactions on Intelligent Transportation Systems, vol. 18, no. 5, pp. 1066-1077, 2017.

[9] Y. Li, K. Li, T. Zheng, X. Hu, H. Feng, and Y. Li, "Evaluating the performance of vehicular platoon control under different network topologies of initial states," Physica A: Statistical Mechanics and its Applications, vol. 450, pp. 359-368, 2016. 
[10] S. Tak, S. Kim, and H. Yeo, "A Study on the Traffic Predictive Cruise Control Strategy With Downstream Traffic Information," IEEE Transactions on Intelligent Transportation Systems, vol. 17, no. 7, pp. 1932-1943, 2016.

[11] M. Wang, W. Daamen, S. P. Hoogendoorn, and B. van Arem, "Rolling horizon control framework for driver assistance systems. Part I: mathematical formulation and non-cooperative systems," Transportation Research Part C: Emerging Technologies, vol. 40, pp. 271-289, 2014.

[12] F. Michaud, P. Lepage, P. Frenette, D. Létourneau, and N. Gaubert, "Coordinated maneuvering of automated vehicles in platoons," IEEE Transactions on Intelligent Transportation Systems, vol. 7, no. 4, pp. 437-446, 2006.

[13] V. Milanes, S. E. Shladover, J. Spring, C. Nowakowski, H. Kawazoe, and M. Nakamura, "Cooperative adaptive cruise control in real traffic situations," IEEE Transactions on Intelligent Transportation Systems, vol. 15, no. 1, pp. 296-305, 2014.

[14] Y. Li, L. Zhang, H. Zheng et al., "Nonlane-Discipline-Based CarFollowing Model for Electric Vehicles in Transportation-CyberPhysical Systems," IEEE Transactions on Intelligent Transportation Systems, vol. 19, no. 1, pp. 38-47, 2018.

[15] J. I. Ge and G. Orosz, "Dynamics of connected vehicle systems with delayed acceleration feedback," Transportation Research Part C: Emerging Technologies, vol. 46, pp. 46-64, 2014.

[16] M. Wang, S. P. Hoogendoorn, W. Daamen, B. van Arem, B. Shyrokau, and R. Happee, "Delay-compensating strategy to enhance string stability of adaptive cruise controlled vehicles," Transportmetrica B, pp. 1-19, 2016.

[17] L.-Y. Xiao and F. Gao, "Effect of information delay on string stability of platoon of automated vehicles under typical information frameworks," Journal of Central South University of Technology (English Edition), vol. 17, no. 6, pp. 1271-1278, 2010.

[18] S. Öncü, J. Ploeg, N. Van De Wouw, and H. Nijmeijer, "Cooperative adaptive cruise control: Network-aware analysis of string stability," IEEE Transactions on Intelligent Transportation Systems, vol. 15, no. 4, pp. 1527-1537, 2014.

[19] J. Ryu and J. C. Gerdes, "Integrating inertial sensors with Global Positioning System (GPS) for vehicle dynamics control," Journal of Dynamic Systems, Measurement, and Control, vol. 126, no. 2, pp. 243-254, 2004.

[20] M. Wang, W. Daamen, S. P. Hoogendoorn, and B. van Arem, "Rolling horizon control framework for driver assistance systems. Part II: Cooperative sensing and cooperative control," Transportation Research Part C: Emerging Technologies, vol. 40, pp. 290-311, 2014.

[21] J. Guo, Y. Luo, and K. Li, "Adaptive fuzzy sliding mode control for coordinated longitudinal and lateral motions of multiple autonomous vehicles in a platoon," Science China Technological Sciences, vol. 60, no. 4, pp. 576-586, 2017.

[22] Y. Li, B. Yang, T. Zheng, Y. Li, M. Cui, and S. Peeta, "ExtendedState-Observer-Based Double-Loop Integral Sliding-Mode Control of Electronic Throttle Valve," IEEE Transactions on Intelligent Transportation Systems, vol. 16, no. 5, pp. 2501-2510, 2015.

[23] F. Gao, S. E. Li, Y. Zheng, and D. Kum, "Robust control of heterogeneous vehicular platoon with uncertain dynamics and communication delay," IET Intelligent Transport Systems, vol. 10, no. 7, pp. 503-513, 2016.

[24] S. Sheikholeslam and C. A. Desoer, "Longitudinal control of a platoon of vehicles with no communication of lead vehicle information: a system level study," IEEE Transactions on Vehicular Technology, vol. 42, no. 4, pp. 546-554, 1993.
[25] C.-Y. Liang and H. Peng, "Optimal adaptive cruise control with guaranteed string stability," Vehicle System Dynamics, vol. 32, no. 4, pp. 313-330, 1999.

[26] W. Schakel, V. Knoop, and B. Van Arem, "Integrated lane change model with relaxation and synchronization," Transportation Research Record, vol. 2316, pp. 47-57, 2012.

[27] M. Treiber, A. Hennecke, and D. Helbing, "Congested traffic states in empirical observations and microscopic simulations," Physical Review E: Statistical, Nonlinear, and Soft Matter Physics, vol. 62, no. 2, pp. 1805-1824, 2000.

[28] D. Jia and D. Ngoduy, "Platoon based cooperative driving model with consideration of realistic inter-vehicle communication," Transportation Research Part C: Emerging Technologies, vol. 68, pp. 245-264, 2016.

[29] R. Rajamani and S. E. Shladover, "Experimental comparative study of autonomous and co-operative vehicle-follower control systems," Transportation Research Part C: Emerging Technologies, vol. 9, no. 1, pp. 15-31, 2001.

[30] M. V. Kothare, V. Balakrishnan, and M. Morari, "Robust constrained model predictive control using linear matrix inequalities," Automatica, vol. 32, no. 10, pp. 1361-1379, 1996.

[31] Z. Duan, J. Zhang, C. Zhang, and E. Mosca, "Robust H2 and Ho filtering for uncertain linear systems," Automatica, vol. 42, no. 11, pp. 1919-1926, 2006.

[32] P. T. Boggs and J. W. Tolle, "Sequential Quadratic Programming," Acta Numerica, vol. 4, pp. 1-51, 1995.

[33] L. Xiao and F. Gao, "Practical string stability of platoon of adaptive cruise control vehicles," IEEE Transactions on Intelligent Transportation Systems, vol. 12, no. 4, pp. 1184-1194, 2011.

[34] J. L. Garriga and M. Soroush, "Model predictive control tuning methods: a review," Industrial \& Engineering Chemistry Research, vol. 49, no. 8, pp. 3505-3515, 2010. 


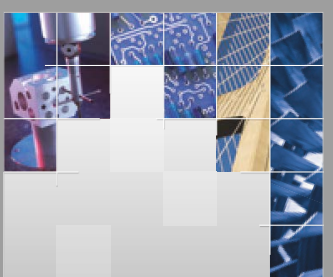

\section{Enfincering}
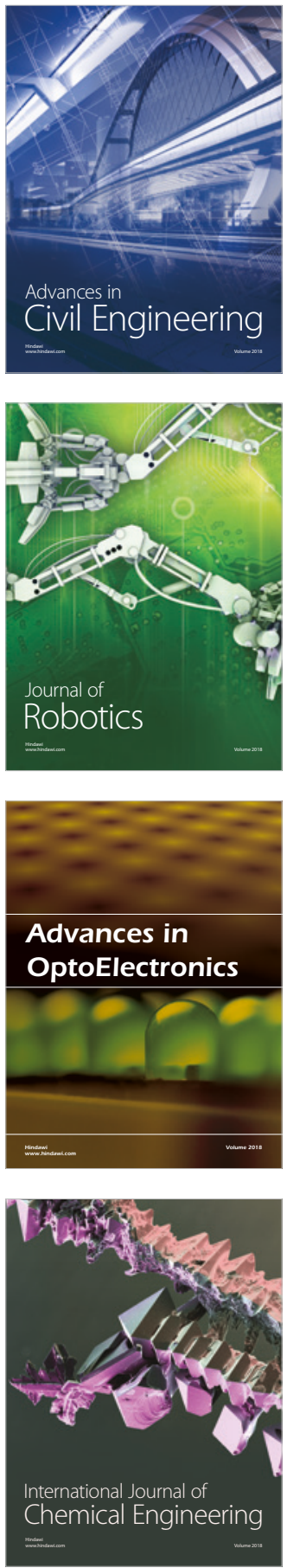

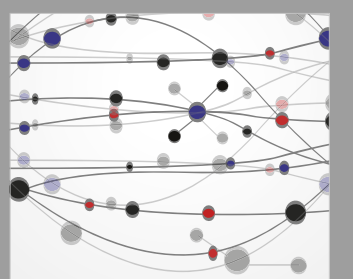

\section{Rotating \\ Machinery}

The Scientific World Journal

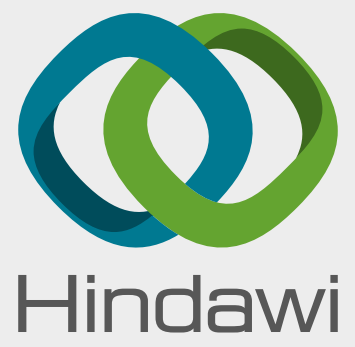

Submit your manuscripts at

www.hindawi.com
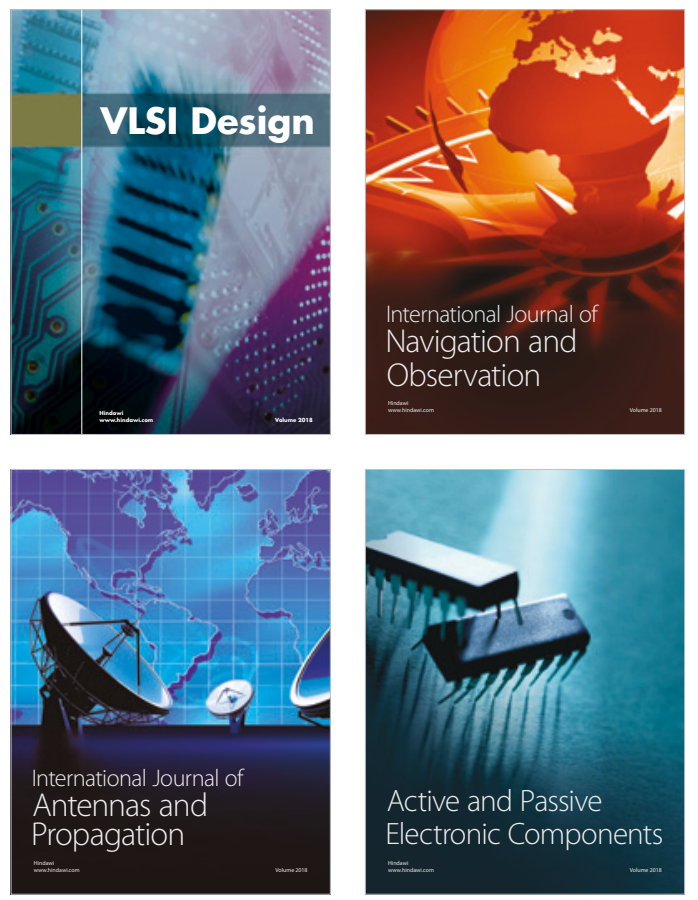
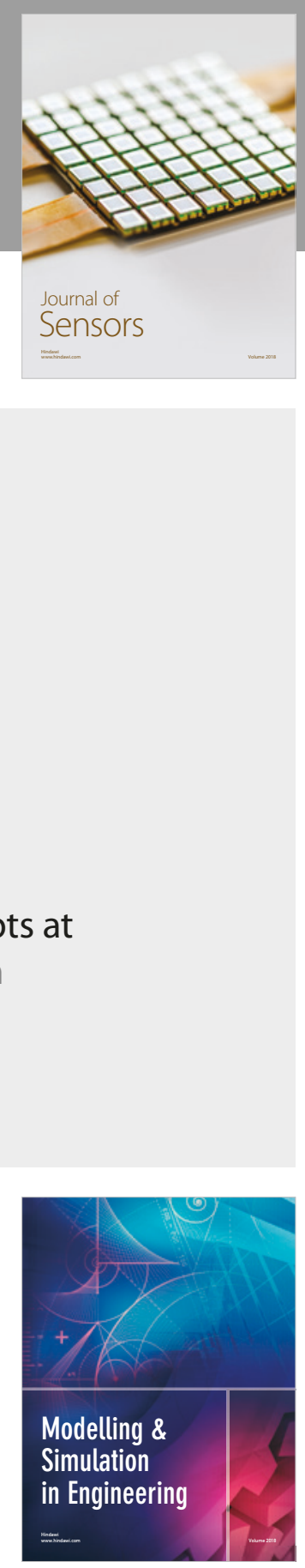

\section{Advances \\ Multimedia}
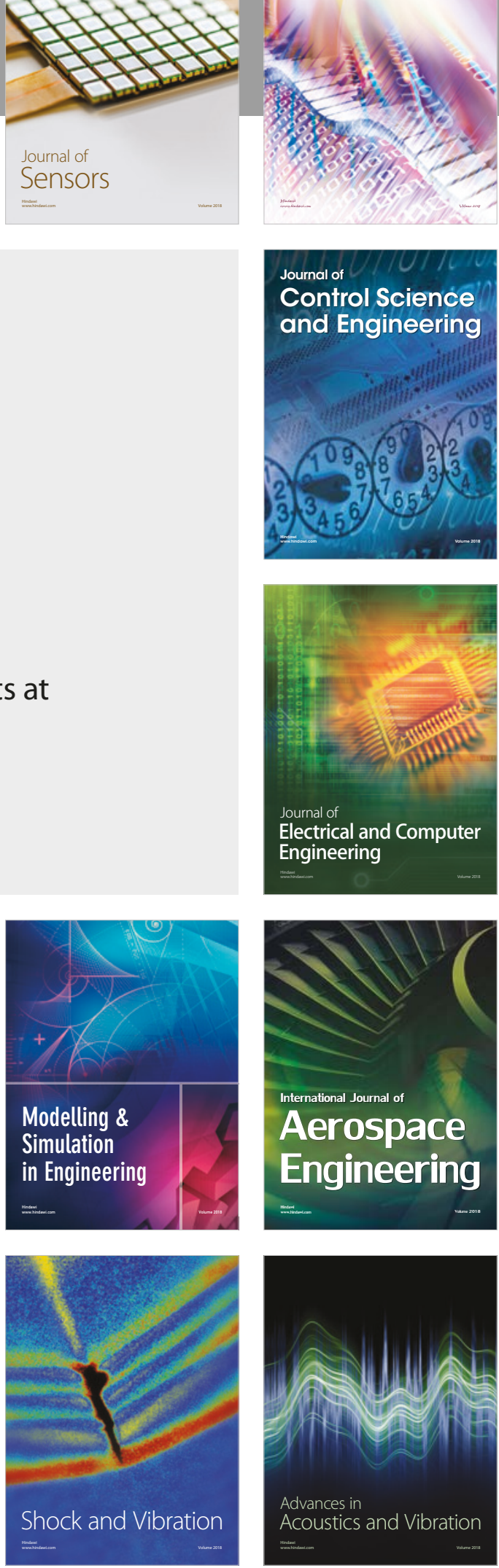